\title{
Novel theory of the human brain: information- commutation basis of architecture and principles of operation
}

\section{Andrey S Bryukhovetskiy \\ Center for Biomedical Technologies, Federal Research and Clinical Center for Specialized Types of Medical Assistance and Medical Technologies of the Federal Medical Biological Agency, NeuroVita Clinic of Interventional and Restorative Neurology and Therapy, \\ Moscow, Russia}

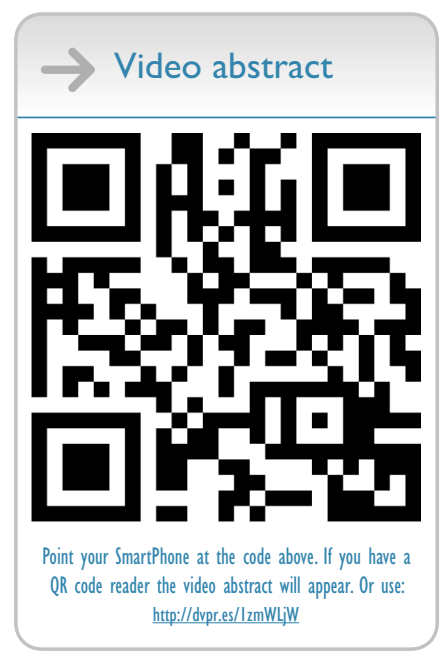

Correspondence: Andrey S Bryukhovetskiy

NeuroVita Clinic, 23 Kashirskoye Shosse, Moscow I I5478, Russia

Tel +74993249339 ext 102

Fax +7495980 ।373

Email neurovitaclinic@gmail.com

This article was published in the following Dove Press journal:

Journal of Neurorestoratology

19 February 2015

Number of times this article has been viewed

\begin{abstract}
Based on the methodology of the informational approach and research of the genome, proteome, and complete transcriptome profiles of different cells in the nervous tissue of the human brain, the author proposes a new theory of information-commutation organization and architecture of the human brain which is an alternative to the conventional systemic connective morphofunctional paradigm of the brain framework. Informational principles of brain operation are defined: the modular principle, holographic principle, principle of systematicity of vertical commutative connection and complexity of horizontal commutative connection, regulatory principle, relay principle, modulation principle, "illumination" principle, principle of personalized memory and intellect, and principle of low energy consumption. The author demonstrates that the cortex functions only as a switchboard and router of information, while information is processed outside the nervous tissue of the brain in the intermeningeal space. The main structural element of information-commutation in the brain is not the neuron, but information-commutation modules that are subdivided into receiver modules, transmitter modules, and subscriber modules, forming a vertical architecture of nervous tissue in the brain as information lines and information channels, and a horizontal architecture as central, intermediate, and peripheral information-commutation platforms. Information in information-commutation modules is transferred by means of the carriers that are characteristic to the specific information level from inductome to genome, transcriptome, proteome, metabolome, secretome, and magnetome. In the brain, the pia mater is an information carrier and the arachnoid is a system administrator and a software carrier. Horizontal commutation between different parts of the brain proceeds extraneurally in holograms formed by the interference and diffraction of reference and object electromagnetic waves of the information-commutation modules of the brain cortex in cerebrospinal fluid. Vertical commutation of the information-commutation modules of various information-commutation platforms is achieved by package impulse transfer of current along axons, and horizontal commutation is performed biochemically by secretomes of cells and synapses in the nervous tissue of the brain. Practical application of this novel theory is demonstrated in an explanation of the pathogenesis and clinical symptoms of several nervous system and psychiatric diseases.
\end{abstract}

Keywords: brain theory, information-commutation theory, alternative brain theory, understanding the brain, brain structure

\section{Introduction}

"Understanding the human brain is one of the greatest challenges facing 21 st century science."1 It is not the anatomical structure or neurophysiological processes of the brain that require understanding, but the basics of human brain structure and its operation and the biophysical and biochemical fundamentals of formation of memories, cognition, and intellect. Understanding of the brain will permit unique neurotechnologies 
to maintain and control brain functions, methods to expand memory and intellect, therapies of nervous and mental diseases, new generation of neuromorphic energy-saving computing systems, artificial brain, artificial intellect and neurorobotics. $^{1-4}$

The goals of this work are to describe an alternative information-communicative theory of organization of the brain and the information principles of its operation, to demonstrate its scientific and practical meaning for neurology, neurosciences, and brain modeling, and to explain the mechanisms of formation and loss of reflexes, consciousness, memories, and intellect in the disordered information structure of brain.

Conventional scientific concepts of brain organization and structure can be summarized as follows:

1. The brain is a multilevel, multifunctional structure in the central nervous system (CNS) of mammalian (including human) organisms intended to receive, transfer, process, and store information received from the body's organs, systems, and environment. The CNS regulates and maintains homeostasis in the organism, promoting its survival and adaptation under constantly changing conditions and extreme environmental factors. , $^{2,3-5-8}$

2. A number of systemic morphofunctional levels of the brain and CNS can be distinguished, ie, the genome, transcriptome, proteome, and metabolome levels, synaptic level, level of the cell, level of tissue circuits, level of brain regions, level of connectivity, level of the whole brain, and the cognitive level. ${ }^{1}$

3. Nerve cells (neurons) are the basic informational structural and functional units of the brain that, being connected through multiple synapses, form neural circuits ${ }^{2}$ providing a morphologic substrate for functional systems in the brain., ${ }^{9,10}$ Organized into neural circuits, neurons have strict topical localization and functional specialization (eg, motor, visual, proprioceptive) in the CNS and cannot be restored if injured. ${ }^{4}$

4. Inside the neuron, information is transmitted by electrical package-impulse transfer through the nodes of Ranvier on its axon, while between the neurons information is transmitted chemically through neuromediators, growth factors, and neurotransmitters. ${ }^{6,11,12}$ Anatomic structures connecting neurons are called synapses and provide a morphological substrate for formation of neural networks. ${ }^{1,2}$

5. Neural circuits are the basic information structures of the brain, and consist of neurons and synapses that support the basic functions of life in a human/mammal, and record and store memory, cognition, and intellectual-mnestic activity. ${ }^{13,14}$ The cerebral cortex can be perceived as an information system consisting of hierarchical groups of neural circuits. ${ }^{15}$

6. Overall, the brain is a multilevel neural network ${ }^{16,17}$ that receives, processes, and stores information which is evenly distributed among all neurons of the brain due to the holographic principle. ${ }^{10,18}$ At the same time, the human brain can also be represented as a hologram capable of forming different functional systems to satisfy the needs of the organism and to achieve a useful adaptive result. ${ }^{9,10}$

7. Memory is one of the basic functions of the brain, is evenly distributed among all cells of brain cortex and subcortical nodes, and is stored in the protein and genome networks of neurons, ${ }^{19}$ permitting recording, storage, and reproduction of the information in every region of this multilayered neural network. ${ }^{20,21}$ The main substrate of the brain responsible for memories is the hippocampus, activation of which by optogenetically manipulating memory engram-bearing cells can result in false memories. ${ }^{22}$

8. Morphofunctional systems in the brain are based on circuits providing functional cortical neurodynamic integration ${ }^{23-25}$ of different brain regions and brain formations and this is manifested in bioelectrical activity, oscillatory processes, ${ }^{26}$ and magnetoencephalographic manifestations of brain functioning.

9. Neurodynamic integration forms cognitive functions of neural circuits of brain cortex ${ }^{27}$ based on the principles of free energy. ${ }^{28-30}$ Perception and thinking are also the circuit functions; it is the cooperation of different brain areas that keep constantly adapting on the basis of the solved tasks, internal resources of the brain and biological limitations. ${ }^{31}$

10. Information is processed in the brain cortex at the level of neural $\operatorname{codes}^{32-34}$ by activation of the membrane currents of the cortex, as well as simultaneous interaction of different cell levels of the cortex ${ }^{35}$ which is manifested in cognitive functions. Quantum mechanisms of consciousness formation ${ }^{36-38}$ and neuroconnectivity of cerebral cortex underlie the information processing. ${ }^{39,40}$

This summary of the available academic literature on the brain describes a contemporary paradigm of brain operation in fundamental neuroscience and neurology, and these principles underlie all mathematical and neurobiological brain simulations.

The numbers of neurons and synapses in the most advanced models of artificial intelligence are very similar to those in the brain, as reported in Stewart et al, ${ }^{41}$ the IBM Sequoia, ${ }^{42}$ the Blue Brain, ${ }^{2}$ and others, who have visualized 
the microstructure of the brain in high definition, as in the artificial intelligence model developed at the Allen Institute for Brain Science (Seattle, WA, USA). However, it appears that copying the number of neurons and synapses does not allow even $1 \%$ of common human brain functions.

Supposedly, the explanation for this lies in our stereotypical, conservative, and physiology-based thinking, which consequently requires revision. Primarily, we need to revise our understanding of the role of the neuron in the nervous tissue (NT) of the brain and in the information-communicative processes of cognition, as well as to understand the informational meaning of a range of neuroanatomical formations closely related to brain. Their informational and communicative functions in the brain have to be reviewed from the standpoint of "theoretical" neurology (similarly to theoretical physics) and available concepts of information science (informationology), and not from anatomical, neurological, and morphological points of view. Our predecessors, including neuromorphologists, neurophysiologists, and neurologists, perceived the main functions of "accessory" structures of the head (pia, dura, arachnoid, cerebrospinal fluid [CSF], skull) to be mechanistic and supporting or physiological.

The other problem lies in the fact that neurosciences develop empirically, and not by development of scientific theories, hypotheses, and concepts, as happens in the exact science. The absence of brain theory and even a framework of ideas regarding the operation and structure of the brain was mentioned by Francis Crick, the Nobel prize winner, in September, $1979 .{ }^{43}$

Informationology (the science of reception, storage, and processing of information) considers a process to be informational if it occurs due to research of the interaction and interrelationship of elementary particles, micro bodies, and macro bodies (Neumark). ${ }^{44}$ The unit of an elementary relationship is known as an "information bit" which is, in fact, a general physical elementary quantum of the relationship between micro dynamic and macro dynamic processes and phenomena. Information interactions yield physical information fields, the constant intensity of which is supported by resonant-cellular, frequency-quantum, and wave oscillation of molecules, atoms, and elementary particles. All interactions between elementary particles, atoms, molecules, cells, tissues, and body organs begin with field interaction, ie, communication is carried out at the informational level of fields of all types, their traces, and all spin-spin, virtual, hypothetical, vacuum, and other forms of interactions at the micro and macro levels. The information consists of principal links (eg, between fields, spins, and their traces) that can be revealed as fluctuations of (related) particles, frequencies, electrons, photons, resonances, oscillations, and prompt emissions. Information manifests as electromagnetic, gravitational, and torsion (spin) fields. The fields create relationships, correlations, interrelationships, interdependencies, and interactions between massless and mass elementary, virtual, and hypothetical particles which, according to the information code, determine their long existence or prompt decay. Interaction of information bits establishes an informationcellular space, which is wave and resonant; it oscillates and fluctuates relative to the equilibrium state of electrons. The state represents a "standing" wave with its own resonant frequency, being a resonant oscillation of electrons, atoms, and bodies. A standing wave of elementary particles is characterized by the interference when electromagnetic waves, being constantly reflected in space, interfere and create standing waves with corresponding oscillation of electrons.

Jeff Hawkins, head of the Redwood Center for Theoretical Neuroscience (Berkeley, CA, USA) quotes Francis Crick by saying "We are in the pre-paradigm days, if you want to use Thomas Kuhn". ${ }^{45}$ According to Dr Hawkins, “... the great bulk of knowledge in neuroscience has been recently generated but we do not even know how to begin to organize it. This is actually a symptom of the fact that we don't have a theory." 45

Another methodological error in brain research lies in the use of a systemic approach to the analysis and processing of neuroresearch data. The revolutionary methodology of the 20th century resulted in many scientific and technological breakthroughs; however, in the 21 st century, the systemic approach hinders our understanding of the brain, and makes us stick to the details, such as, neural, protein, and gene networks, and logical graph structures and trees.

To illustrate the above comments, the methodology of the Human Brain Project links objective information (genome, transcriptome, proteome, metabolome) with subjective notions (cognitive functions, connectome) and neural and morphological phenomena (cells, synapses, region of the brain, the whole brain). Moreover, biophysical data on the brain and their correlations with biochemical data are not considered, and researchers plan to develop information and communicative platforms and neurotechnologies without a conceptual framework of information theory, which seems technically incorrect.

The goal of this work is to describe the brain as an information-communicative device and its principles of work, to demonstrate its scientific and practical meaning for neurology, neurosciences, brain modeling and to explain the mechanisms of formation and loss of reflexes, consciousness, 
memories and thinking in disordered information structure of brain.

\section{Justification of the theory}

It would seem that the anatomical structure of the brain should not be viewed only from the positions of neurobiology, neurophysiology, and neuropsychology, but also evaluated from a methodological standpoint, for example, by information theory. Further, the methodology of the systemic approach should be replaced with the methodology of information theory. Here an attempt is made to extrapolate the fundamentals of information theory on the human brain. In this respect, the following is noteworthy. First, information cannot be transferred on its own without carriers of material information. Therefore, the key task is to understand how information is formed and transformed, and which material carriers it engages. The information about the body and the environment is usually delivered by electromagnetic waves (EMWs) to the receptors of sense organs and, accordingly, changes their field characteristics and general magnetization of the cell. The information from the EMWs is transferred, embeds into electromagnetic field of the cell and changes it accordingly. The change of the cell magnetization and cell electromagnetic field at the level of its inductome by the input information leads to the change in the state of some DNAs in the genome. The information transfers to these DNAs. That is, at the level of the cell genome these separate DNAs are the carriers of the information, they produce specific microRNAs; and the information further is transferred by them. At the level of cell transcriptome the main carrier of the information is RNA. Different RNAs (that were activated by the information) change the cell proteome and its phenotype. Therefore, at the level of cell proteome the information is carried by the proteins, that transfer information to the lipids and carbohydrates at the level of metabolome and it is further used for cell metabolism. At the level of cell secretome, the mediators, neurotrophins, hormones and growth factors become the information carriers. The information by means of the secretome provides for biochemical interaction between the cells in the tissue. The other external biophysical transfer of the cell information inside the tissue is general electromagnetic field of a cell. Electromagnetic waves of different cells of nervous tissue interact on the body of a neuron and as a result an electromagnetic impulse of current develops. It follows along its axon through the nodes of Ranvier and quickly transmits the received data from one tissue module to the other on the body of its system-forming neuron. Thus, the data received by the body on the one side trigger the work of all informational levels of the target cell of the receptor, and, on the other side, provide for intercellular interaction, synchronization and targeted transfer of the information inside the tissue. The information changes its carriers inside the cells and, thus, it modulates (strengthens or reduces) depending on its significance for the cells, while preserving its nature. Second, we know that the information structure of an intact cell is a rigid interrelated hierarchy of information levels (genome, transcriptome, proteome, metabolome), ${ }^{1}$ where the slightest changes in genome information level cause programmed changes at all information structure levels. Meanwhile, the information structure at the molecular information level of a cell reflects only its biochemical machinery; it shows how information moves at molecular information levels and we can follow the dynamics of its biochemical processes. This would be appropriate if we studied cells as a complex biochemical structure only from molecular positions of systemic theory. However, the cells in the NT represent a living system where information processes at the resonant wave level, elementary particle level, and atomic level form magnetic fields that control the genome by, eg, frequency resonance, movement of atoms, and oscillations, while at molecular level the biochemical work of the cell is transformed into electric currents of NT. Thus, information is sent to the gene level from the level of field formations and is then transferred to the genome by an information carrier as a magnetic field formed by movements of atoms and elementary particles, molecular resonances, and oscillations. The degree of magnetization and inductance of the tissue characterize its magnetic fields, which can be caused by: ion currents from electrical activity of cell membranes; the finest ferromagnetic particles introduced into the organism voluntarily or involuntarily; and when an external magnetic field is superimposed, the heterogeneity of magnetic susceptibility of different organs manifests and distorts the imposed external field. Similar to other cell information levels, we refer to the magnetic field information level of cell induction that precedes the genome as an inductome. Further, the information "mounts" biochemical carriers (eg, DNA, RNA, proteins), comes through all biochemical cycle of transfer along intracellular structures of molecular level to change for biophysical carriers, and namely, cellular electromagnetic waves. The level of cellular electromagnetic waves is referred to as the magnetome, following the already known names (eg, genome, proteome). The information levels of the magnetome and inductome suppose that a cell system is involved in formation of systems tissue, and its organization and regulation (Figure 1). Obviously, the inductome of a NT cell displays the degree and characteristics of magnetization of a specific tissue and is a controlling value of the field information action on the intracellular 


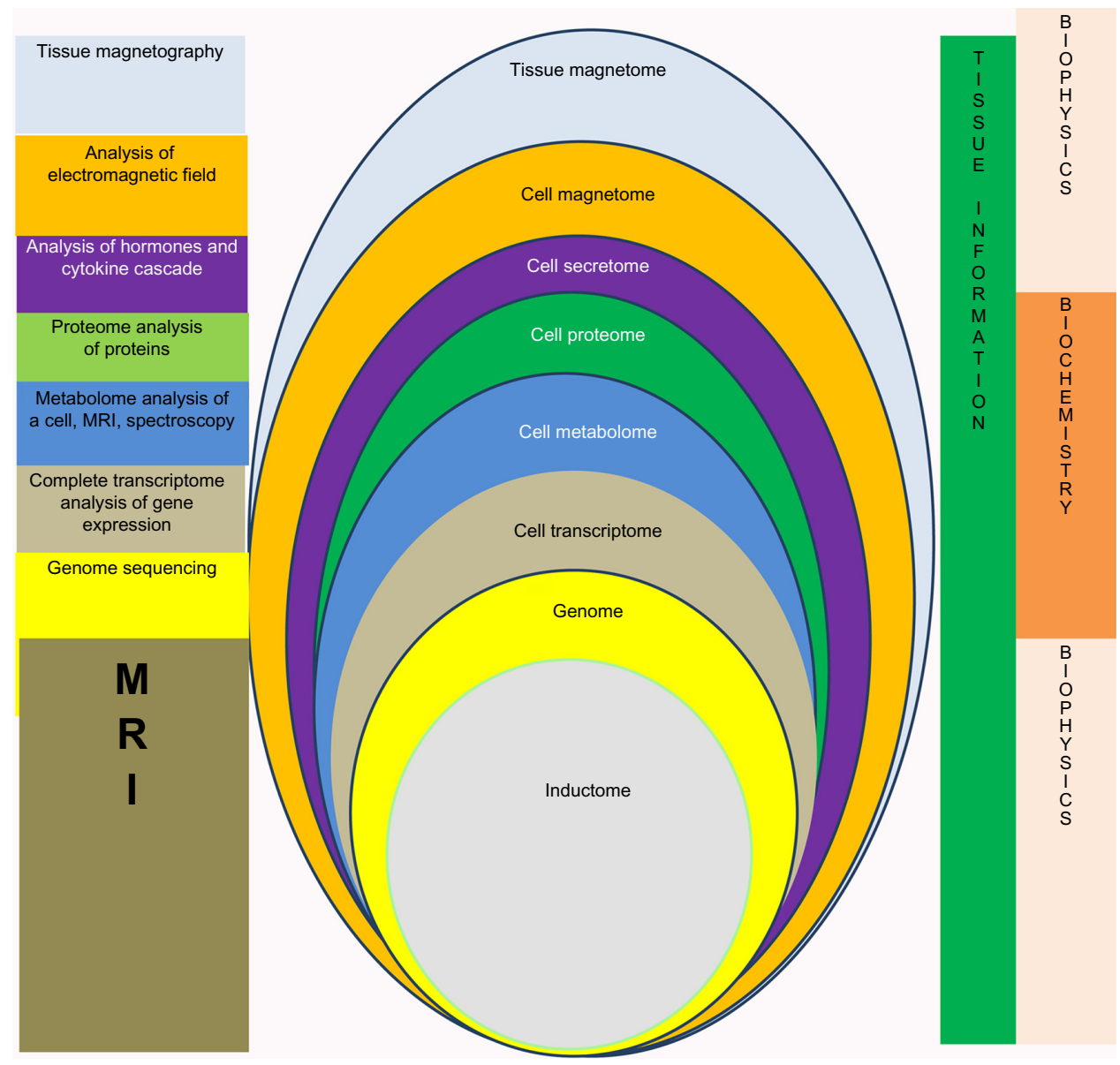

Figure I Complete information structure of nervous tissue and the methods to register it. Abbreviation: MRI, magnetic resonance imaging.

genome; while the cell magnetome provides cell participation in the formation of tissue and organ levels manifest in electromagnetic waves of super high frequencies and can be registered by magnetoencephalography. The work of Devyatkov et $\mathrm{al}^{46}$ showed that the cell magnetome is characterized by electromagnetic waves of super high frequency and extremely high frequency at the cell level, ie, electromagnetic waves in the millimeter range. The inductome in brain tissue can be registered with magnetic resonance imaging of the brain.

Information sectors of NT cell systems represented as magnetomes interact and form a general database of specific NT information-communication modules (ICMs). In living systems the cells integrate in tissue, and the tissue information level develops due to interaction of cell sectors of information structures; this interaction primarily happens at the level of interaction of their magnetomes, thereby forming a tissue magnetome that is an integral parameter of the composition and imposition of electromagnetic wave magnetomes of different NT cells (eg, oligodendrocytes, astrocytes, microglia). The information basis of the tissue has a controlling influence on organization and survival of specific NT ICMs and on generation of organs from various tissue modules. Integrating with each other, electromagnetic waves from different NT cells form electrostatic charges on the bodies of ICM neurons, which can be transferred for large distances inside neurons to other NT ICM through their axons as packages of electric current impulses. Electric currents in neurons generate a magnetic field with $10^{-14}$ to $10^{-11} \mathrm{~T}$ induction that occasionally spreads beyond the organism.

Provisional division of the information structure into information levels of NT ICMs acquires physical significance due to contemporary methods of genomics and post-genome technologies (transcriptomics, proteomics, metabolomics, secretomics), magnetic resonance imaging, and magnetoencephalography. Today we are able to describe and profile each of these information levels of NT ICMs and can present digitalized information for each of these data profiles. When we digitalize every level of NT ICMs, the virtual structure becomes real (Figure 2). Here we mean the information structure of a whole tissue, as modern technologies cannot evaluate the information structure of a single cell; at least 1.5 or 2 million of NT cells are needed to profile proteins in the cell proteome, to analyze the DNA 


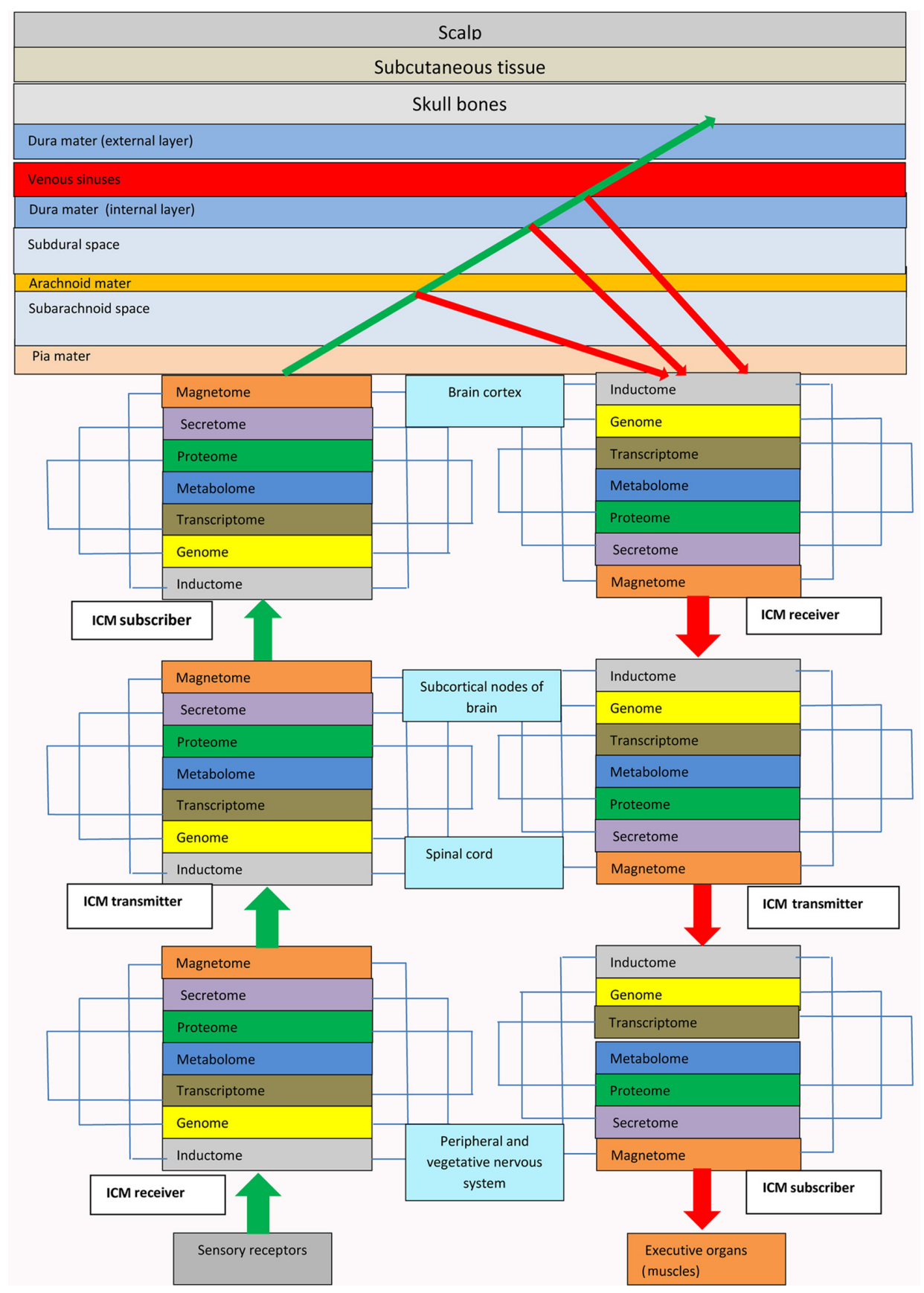

Figure 2 Extraneural mechanism of reflex activity of the brain.

Abbreviation: ICM, information-communication module.

profile at the genome level, and to study the RNA profile at the transcriptome level.

\section{Principles of human brain operation}

Relying on genomic, transcriptomic, and proteomic research regarding NT cells in health and disease $\mathrm{e}^{8,47-48}$ and our work in theoretical neurology, we have developed the following information principles of brain operation.

\section{Modular principle}

The NT ICM, and not the NT neuron, is the backbone communicative-organizing information unit in the human brain. It exists as a self-regulating multilevel information structure, which is morphologically specific to its location in brain. The main functions of the NT ICM are reception, transfer, accumulation, transformation, and modulation (upregulation or downregulation, additional generation) of information. NT ICMs 
do not participate in the processing, analysis, or synthesis of information. Depending on their functional assignment and role in information transfer, they are subdivided into NT ICM receivers, NT ICM transmitters, and NT ICM subscribers. The modules form information output and input lines that in turn form appropriate information output and input channels for information reception and transfer.

\section{Holographic principle}

In the brain, information is processed according to the principle of holography, although not in the NT, but beyond the brain parenchyma. Cortical ICM subscribers form electromagnetic waves that carry information in electrostatic charges via information input channels and information lines from different brain analyzers. Electromagnetic waves leave ICM receivers of information input channels in the brain cortex and forward to CSF in the subarachnoid space (SAS) and subdural space (SDS). CSF scatters electromagnetic waves and forms object and reference electromagnetic waves. Reflected from the arachnoid and dura, the object and reference electromagnetic waves interact (Figure 3). Interference, diffraction, imposition, and composition of the object and reference electromagnetic waves and the reflected electromagnetic waves in the SDS result in holograms that become a new integral information carrier in the SAS and SDS (Figure 4). Therefore, the information is processed in the SAS extraneurally and not in the NT of the human brain. The SAS in the brain is a space where reflex arcs of ICM electromagnetic waves in information input channels commute with ICM electromagnetic waves of information output channels of these reflexes. The SDS in the brain is a space where holograms of the information image matrices (IIMs) commute to different brain areas. SAS and SDS function as processors for a biological supercomputer in the brain. The holograms are transported by the CSF over a large area on the brain surface and their electromagnetic waves passing through the arachnoid leave traces (engrams) of IIMs on the arachnoid. The arachnoid in the course of human life
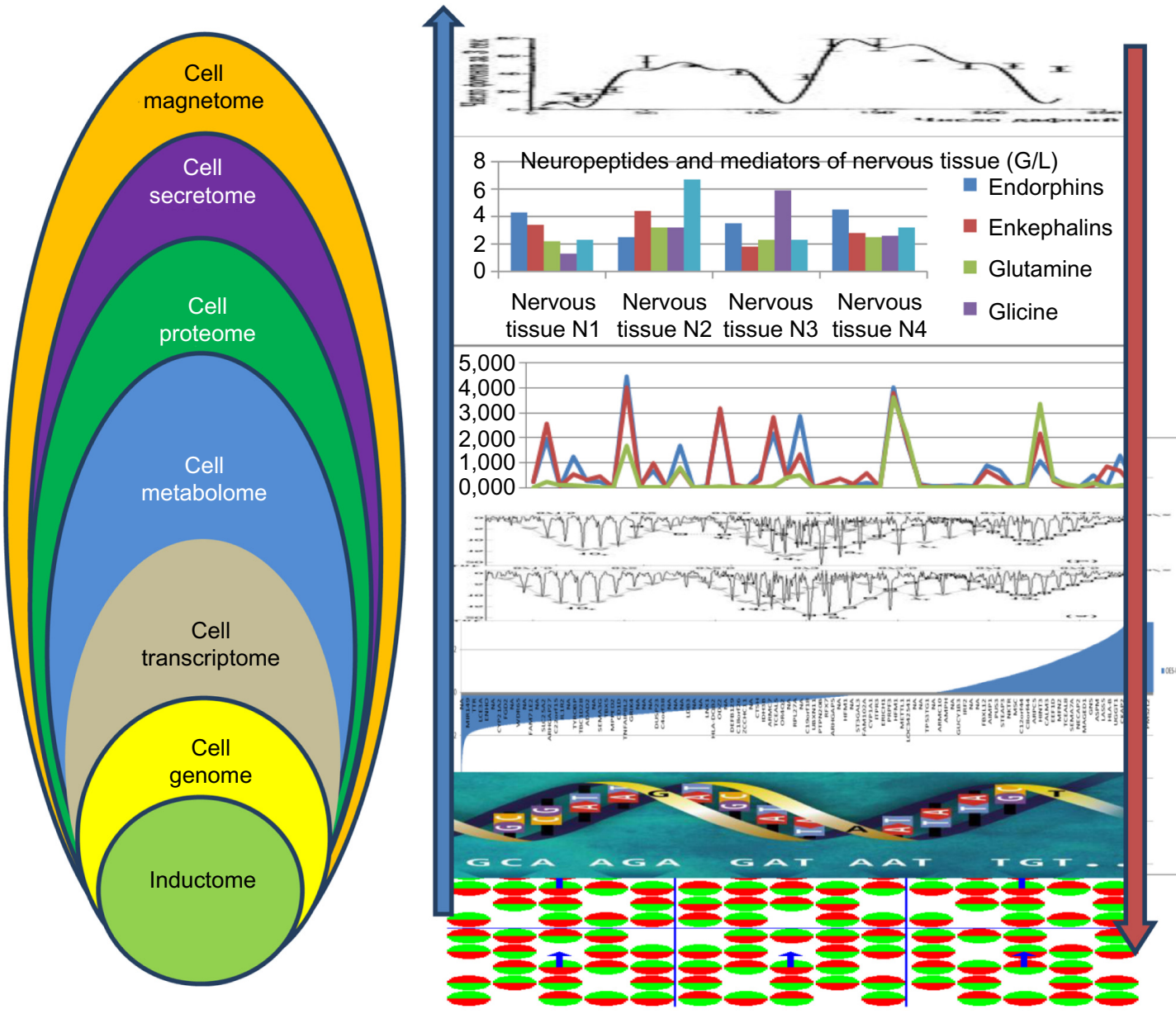

Figure 3 Multilevel mapping and profiling of information structure of the somatic tissue. 


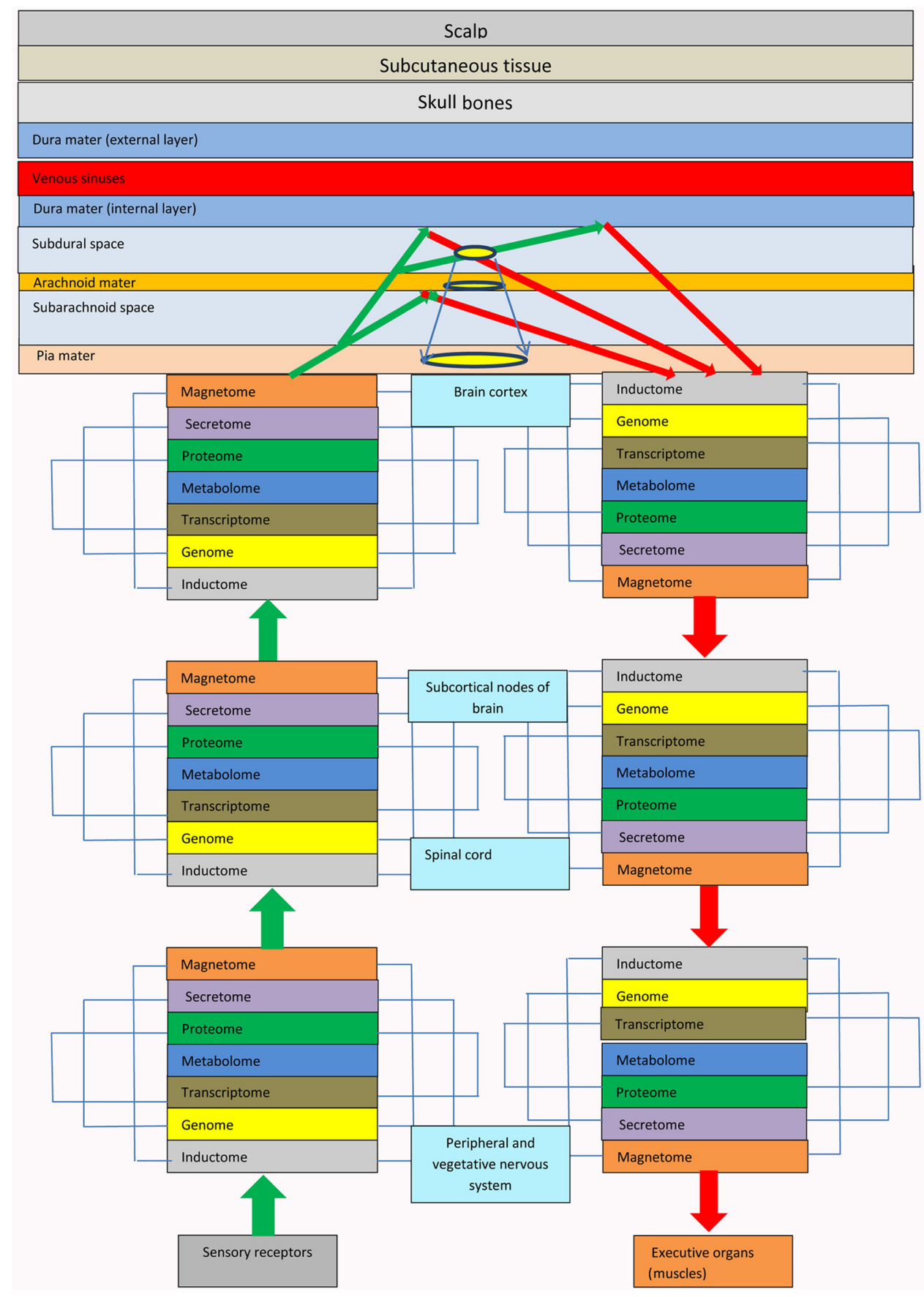

Figure 4 Extraneural mechanism of intellectual control of reflex activity of the brain. Note: Yellow oval with blue frame denotes hologram.

and acquisition of experience becomes a software punch card located over the brain. Holograms are transferred by natural CSF flow due to the physiological pulse of the brain (6- 8 beats per minute).

\section{Principle of systematicity of vertical and complexity of horizontal commutative connections}

The brain has two types of information-commutative connections, ie, system vertical commutation and complex horizontal commutation. In system vertical commutation of information, NT in the brain functions as a telephone switchboard, dispatcher, and router of information through NT modules for information input and output channels and information lines. Vertical system commutation inside ICMs and between an information communication platform that consists of ICMs is performed only in one direction of interlevel interaction in NT ICM: from the cell inductome to the genome, transcriptome, proteome, metabolome, and magnetome and then to the tissue magnetome. Vertical 
commutation between ICMs is performed via packages of impulse currents through the axons of neurons in these ICMs. Thus, rigid systemic directionality (afferents and efferents) of vertical commutation is achieved along information channels and information lines only upwards or downwards between NT ICMs. Horizontal complex commutation of information at different levels of NT ICMs is achieved biochemically and reciprocally through the synapses between nerve cells or by interaction of the cell secretomes included in the structure of the module. Horizontal complex commutation at the SAS and SDS levels is performed by generation of connecting holograms between information input and output lines of various analyzers (eg, motor, visual, auditory, sensory). Hologram formation is a key mechanism of horizontal complex commutation in extraneural connections; holograms are transported by the natural flow of CSF, permitting longdistance transfer of information and commutation between various brain regions.

\section{Regulatory principle}

Information circulates in the brain continuously and cyclically, so that it can maintain the processes of homeostasis and sanogenesis in the brain and body. Independent of the phases of brain function (eg, sleep or wakefulness, excitation or inhibition, stress or rest) the brain never "rests" or "relaxes", and is an example of information perpetuum mobile. Even during sleep, processing does not decrease, and only its routing and commutation connections and properties change. Sleep functions as a switchboard operator of the regulation, commutes information inside each NT ICM, and provides transition from dialog mode of central, intermediate, and peripheral ICM information relationships to an autonomous regulatory functioning mode within an information communication platform. During sleep, ICMs synchronize and regulate their autonomous work and information relationships within the information structure of the tissue, as well as the information structure of each somatic cell. They test the functioning of interlevel information relationships both inside the cell and inside the tissue, comparing them with reference samples in the program files of information sectors of every cell genome, and correct faults and failures. Thus, self-regulation and selfadjustment is provided inside each NT ICM, and if necessary, faults in interlevel and intralevel relationships of NT ICMs can be detected and repaired. This explains the diversity in need for sleep between different people. This is the time necessary for NT ICMs to test their information levels and correct defects. Having detected the defetcs, the responsible for repair cells and tissues activate in the genes of every cell system of tested NT ICMs synchronically, and this activation is accompanied by purposeful changes in magnetic field, transcriptome and proteome cell profiles and correction of the defects. Dreams result from the testing, self-regulation, and self-adjustment of central NT ICMs, and jerks and twitches of the sleeping body and extremities are manifestations of the autonomous activity of peripheral NT ICMs.

\section{Relay principle of commutation}

In the brain, information is a "relay baton" that is continuously transferred unchanged within NT ICM from one level to another. Information is transferred continuously through ascending information channels and information lines between NT ICMs by double-step electromagnetic package transfer from peripheral NT ICMs to intermediate NT ICMs (one step) and from intermediate to central ICMs (two steps), and, respectively, vice versa in descending information channels and information lines. If information is transferred over long distances, it is modulated, coded, and amplified as necessary by static NT ICM transmitters located on the route of information. Interactions and interrelationships between electromagnetic wave magnetomes in different areas of the brain organize and commute cortical NT ICMs as a single central dispatching system, synchronize the work of central NT ICMs, balance their structural changes and interactions, regulate profile parameters of information levels within each NT ICM, thus providing prompt multilevel transition of information through information channels and information lines.

Biophysical integration of NT ICM at the level of magnetomes in neural cells and NT leads to synchronization and a coordinated reaction of various biochemical parameters of information levels downstream (proteome, transcriptome, genome) and to the balance of information between different NT ICMs. The relay principle ensures continuous transfer of information, so that the information only changes carriers and direction.

\section{Principle of modulation}

Modulation (lat. modulatio "rhythmical measure, melody") is the process of varying one or more parameters of the highfrequency carrier wave according to a low-frequency information signal (message). Not dependent on the character, length, or power of the information received by the NT ICM, information is modulated according to its phase, amplitude, and frequency. The controlling (modulating) signal contains the transferred information. A high frequency wave known as a carrier wave plays the role of information carrier, so modulation is the process of "mounting" information on a known carrier (eg, DNA, RNA, proteins, electromagnetic waves, 
holograms) of its information level. It permits adjustment of the functioning of all NT ICM receiving-transferring devices to various frequencies to avoid interference. The principle of modulation of NT ICM organization provides targeted traffic in information channels and information lines. The principle of modulation of brain organization permits registration of the slightest information that can be amplified to be fixable and able to be processed by NT ICM subscribers distant from NT ICM receivers.

\section{"Illuminating" principle}

The idea of an "illuminated" brain cortex was put forward by Francis Crick, the Nobel Prize winner. The term describes the phenomenon very well; it is manifested in activation of a specific cortical area by incoming information. However, it is the pia that is illuminated and not the cortex. Activation and reproduction of memories in the brain are achieved due to the principle of internal and external illumination of mnestic engrams on the pia or contact between the system engrams of the arachnoid and the pia. Holograms of the object are projected onto the pia as IIMs and are recorded on it as on a standard holographic medium in the form of mnestic engrams, and the IIMs are reproduced by illumination (activation) of the engram from below by electromagnetic wave stimuli of the magnetomes of central NT ICM subscribers or by the electromagnetic effect of reflected electromagnetic waves from above. Accordingly, the position of afferent and efferent parts of the cortical terminals of the analyzers that receive excitations from the organism and analogous parts of the analyzers receiving excitation from the environment permits momentary transfer and processing of incoming information and enables an immediate response to it.

\section{Principle of personalized memory and intellect}

A human is born with a clear pia and arachnoid. Accumulation and personalization of memories and IIMs is specific to the individual. Every object, action, and thought generated by the subject has its IIMs in the SAS and SDS registered on the surface of the pia in the course of growth, education, and training, and their number constantly grows with life and experience. This information is registered on the pia and can be reproduced at any time if the "hard disc" of the brain, in particular the pia, is intact. Information recorded on the pia forms personalized and an extremely individual volume of human memory and intellect operating at the information level of consciousness by individual algorithms of the arachnoid similar to "hard drive" inquiries. Personal information received from internal and external sources is registered on the pia automatically and is evenly distributed on the surface of the information carrier. This method to store information is extremely reliable and in case of mechanical or other injuries the information can be restored even by a small fragment of the preserved information carrier.

\section{Principle of low energy consumption}

The use of the mechanism of extracerebral holographic processing of information enables an unlimited number of simultaneous brain operations with no specific energy consumption. It also provides energy-efficient formation of IIMs that does not require additional energy resources to expand the memory capacity for the record, storage and reproduction of the information. Memory resources on the pia do not require energy consumption and the information capacity of the pia, the surface of which is about $2200 \mathrm{~cm}^{2}$, is enough to record the information equivalent to 22000 bibles. The brain uses the pia to record, store, and reproduce information without additional power consumption.

\section{Organization and architecture of the brain}

This research on theoretical neurology and the principles of brain operation has resulted in an alternative informationcommunicative paradigm of brain organization:

1. The brain of a human/mammal is a multilevel modular structure with an open-type architecture of communication and performs the functions of recording, transferring, and routing of information through NT. Programmed by life, training, and education, NT in the brain is a universal switchboard and dispatcher of information received from the environment and from the inner organs and systems of the body. All processes concerning the management, processing, and storage of information are performed extraneurally (outside the NT of the brain) in the intermeningeal CSF information space around the brain between the pia, arachnoid, and dura (Figure 5).

2. The system-forming, assembling, transport, and communicative basis of the brain information organization architecture is the NT ICMs. These are basic bioinformation stations receiving, transferring, modulating, and routing information in the brain and representing a whole complex of basic NT elements (neurons, oligodendrocytes, astrocytes, microglia cells, brain muscle cells, vessels, synapses, neural fibers, and ganglia). NT ICMs, and not separate neurons or neural cells as believed previously, route the information in NT, direct it, and enhance it with the necessary characteristics. The main function of the neurons is network commutation, achieved by forming 


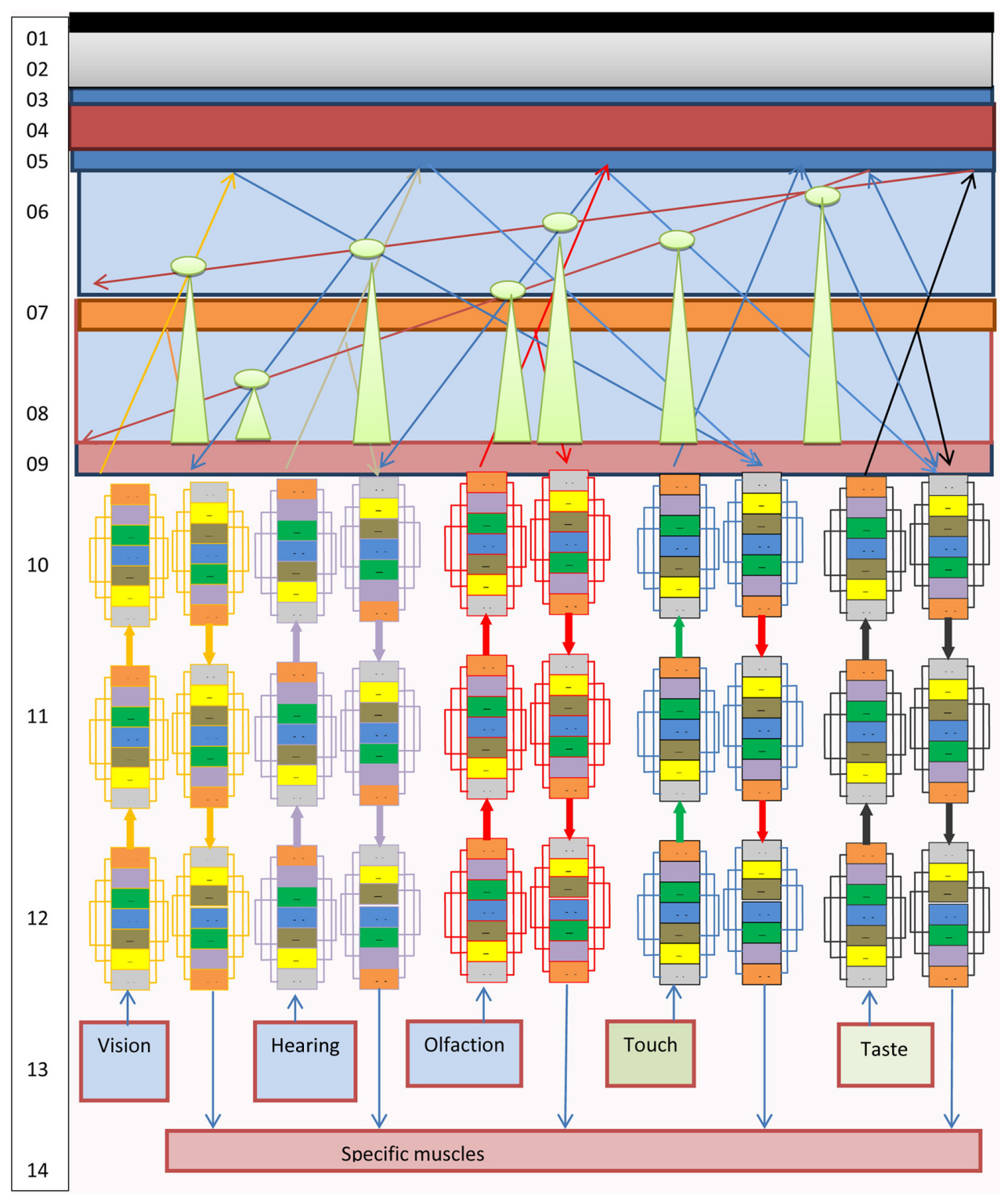

Figure 5 The scheme of brain organization and mechanisms of hologram formation.

Note: The numbers stand for: I. Scalp and subcutaneous tissue; 2 . Skull bones; 3 . External layer of dura mater; 4 . Venous sinus; 5 . Internal layer of dura mater; 6 . Subdural space; 7. Arachnoid mater; 8. Subarachnoid space; 9. Pia mater; I0. Cerebral cortex; II. Subcortical nodes of brain and spinal cord; I2. Peripheral and vegetative nervous system; 13. Sense organs; 14. Executing organs. Light grey - inductome; Bright yellow - genome; Brown - transcriptome; Blue - metabolome; Light purple - secretome; Light orange - magnetome.

neural fibers for conducting pathways to provide connection and interaction between different NT ICMs and rapid targeted intermodular transfer of information. Each NT ICM has a tissue-specific structure that consists of interdependent and interregulated information levels, ie, the inductome, genome, transcriptome, proteome, metabolome, secretome, cell magnetome, and tissue magnetome. Every cell system included into NT ICM structure occupies its own information sector and has ILs analogous to modules. These ILs on the one hand, influence general communicative and conductive information characteristics as well as the information component of a whole module, and, on the other hand, they are exposed to
NT ICM regulatory control. The tissue-organizing factor of NT ICMs in the brain is the level of tissue magnetome that integrates magnetic wave levels of all information sectors of cell systems entering the NT ICM structure. The NT ICM tissue magnetome is formed by the composition, imposition, diffraction, interference, and integration of all super high frequency electromagnetic waves in the cell. Tissue magnetome electromagnetic waves are manifested in the millimeter range of extremely high frequency and form the main control unit for information routing.

3. Central NT ICM subscribers of information input channels and information lines and NT ICM receivers of information output channels and information lines 
form cerebral cortex where they are located as close to each other as possible to provide optimal interaction of the signals of one functional analyzer. Commutation of horizontal information in the brain cortex takes place extraneurally outside the brain parenchyma in the SAS and SDS. Cortical regions integrated by a single function as cytoarchitectonic cortical fields of neighboring brain gyri are biological substrate of brain, thus permitting integration of reflex and cognitive information of central NT ICMs. From the information theory viewpoint, the brain and cerebellar cortex consist of a set of typical NT ICM subscribers of information input channels and NT ICM receivers of information output channels that are equally distributed in the brain gyri, representing a central information communication platform of the brain, which is a control center that coordinates input and output information received from the SAS and SDS. Biologically, active parts in the region of the brain subcortical nodes, spinal cord, and vegetative ganglia of the autonomous nervous system form an intermediate information communication platform that consists of combinations of NT ICM transmitters of both information input and output channels and information lines. The intermediate ICP functions as the main switchboard station for information routing. The neuroreceptor system of the brain (visual, auditory, sensory, motor) is a peripheral registration information communication platform that consists of NT ICM subscribers of information output channels and information lines of the corresponding analyzers. The sympathetic and parasympathetic nervous systems form a peripheral executive information communication platform that consists of NT ICM receivers of information input channels and information lines linked with the nervous-muscular segment of locomotor system and provides motor functions of brain. Central, intermediate, and peripheral information communication platforms control appropriate brain areas, thus establishing a hierarchy of vertical control from the center to the periphery of NT in the brain.

4. The general architecture of brain information structure presupposes novel architectural solution for the brain device which is realized through multi-level informationcommutation platforms. Each of the platforms consists of different informational modules integrated by systemforming morphofunctional factor. Integration of the platform into modules is conditioned by their function and anatomical position in the brain.
5. Each brain information line has its specific information carrier. Any information transferred to the brain by peripheral receptors does not disappear but circulates in the brain continuously and is registered on the main cerebral (genome, proteome, transcriptome) and extracerebral (holograms, pia, arachnoid) material carriers of information in an automatic and distributed fashion. In the intact pia and arachnoid, information is recorded onto these carriers automatically and stored for life (or almost), and can be reproduced when necessary.

6. The arachnoid plays the role of a system administrator, a "system disc" of a biological computer and the reflex activity software in the extraneural ICP of reflex subscribers. It stores all software and registers the order of all algorithms and completed programs of IIM manipulation that are generated during training, education and life of a human. The mechanism of the recording and reproduction of the software algorithms is formed by the electromagnetic waves projection of holograms on the pia. The holograms systematically pass through the arachnoid and leave the traces (engrams) of IIMs on it. The pia plays the role of the "hard disc" in the brain's biocomputer. On the one hand, it is a unique holographic membrane that covers the brain cortex, and on the other hand, it is intensively penetrated by blood vessels that can rapidly transfer information from the organs and vessels to the NT commutative mechanism. Further, the main fibers of the vegetative nervous system enter the pia, thereby enabling control of the vegetative life of the organs, tissues, and vessels (Figure 5).

\section{Discussion}

The principles of brain operation and architecture of information-commutation organization (ICO) described here provide a novel insight into the mechanisms and nature of clinical symptoms of neurological and psychiatric diseases that so far do not have a scientific explanation, as well as into some curious facts concerning operation of the brain. Accordingly, consciousness is extraneural information-commutation connections of different regions of brain NT existing at a specific time and registered on the pia. This novel ICO and the principles of brain operation can explain some known facts of clinical neurology.

To begin with, we examine traumatic brain injury (TBI) and its symptoms from the stance of brain information theory. 
Disordered consciousness or loss of consciousness is a hallmark of acute TBI. But why is consciousness lost in TBI?

Clinical neurology says this is a characteristic symptom in terms of nosology and results from minor micro contusions and micro hemorrhages in the brain caused by minor shock affecting the skull or bones of the spinal column. It is also explained by the coup injury occurring under the site of impact or contrecoup injury on the opposite area associated with cerebral contusions. This seems right, but how can we lose consciousness for a couple of seconds or even a minute in the absence of any pronounced organic change confirmed on magnetic resonance imaging, and then again remember and understand everything? If the reason for this was interneural axonal injury (eg, damage to neural circuits, synapses, and synaptic contacts), consciousness would not be restored in a few moments. Recovery after such injuries takes years and not seconds. However, our understanding of TBI changes if we acknowledge that reflexes and cognition have an extraneural mechanism, ie, at the level of the intermeningeal space, and follow the previously described mechanisms. A shift of the brain in the skull, even by several millimeters, disorders the localization of reflected electromagnetic waves from the NT ICM and the output information lines miss the ICM receivers. So the displacement of the brain leads to short-term disorder of cerebral electromagnetic commutation connections between different regions of the brain and disordered records of the data on the pia, which is accompanied by the loss of consciousness. As soon as the brain restores its location in the skull and its conventional informationcommutation connections between different regions of the brain recover, memory and consciousness recover too. The extraneural commutation of different areas of the brain is disordered and, consequently the active neurodynamic connections are not recorded at the moment of injury and visual, audial, motor or sensory IIMs are not registered on the pia. Thus, we receive memory failure of the injury events. The duration of loss of consciousness is the time taken for the brain to return to its initial position after the injury and to restore commutation connections.

Major disorders of consciousness are directly related to impeded formation of holographic IIMs in the intermeningeal space when it is filled with blood cells that obstruct the permeability of the pia and arachnoid for CSF and influence diffusion of electromagnetic waves. Collision of magnetome electromagnetic waves with blood elements interferes with conditioned and unconditioned reflexes, so that reflexes are depressed in the initial stages of coma and are absent in deep coma. Absence of reflex connection characterized severity of depression of consciousness. Blocking of information at the level of the cortical ICMs causes loss of reflex connections in SAS and absence of IIMs in the SDS in meningitis and meningoencephalitis. Clinical neurology conventionally explains depression and absence of reflexes by disorder of synaptic connections and brain edema, however, our explanation seems to give better answer.

Memory disorder is the key symptom in the clinical picture of both acute and chronic cerebral contusion. The events of retrograde or anterograde amnesia are characteristic for TBI accompanied by micro hemorrhages and micro ischemias in the brain parenchyma as well as edema and swelling of the brain. Current clinical neurology and neurosurgery explain the symptoms of cerebral contusion by reactivity of NT, an inflammatory response to micro injury, swelling of the injured brain, and frequent subarachnoid hemorrhage. To a certain extent this is true, but does not explain the memory disorders seen. According to the proposed brain theory, on the one hand, swelling and edema of NT in the brain blocks information transfer through cortical NT ICMs and exiting of electromagnetic waves into the SAS and SDS, while on the other hand, even slight subarachnoid hemorrhage in acute cerebral contusion affects the transparency of the CSF, and consequently disrupts its information properties due to sedimentation and adhesion of blood elements on the pia, arachnoid, and dura. The disorders of IIMs depend on the number of blood cell elements in the intermeningeal space. Both processes lead to disordered consciousness, such as obnubilation, a soporific state, or even coma, which is conditioned by the loss of reflexes, because the reflex arcs do not connect in the SAS and by the absence of electromagnetic wave holograms in the SDS. Therefore, if the events of the period that preceded the injury were not recorded, they cannot be reproduced and that is how the retrograde amnesia is formed. As soon as the cells in the CSF are lysed (although the CSF remains xanthochromic), consciousness is restored despite high protein levels in the CSF. Blood in the CSF caused by injury leads to brain swelling, ie, to commutation signal failures in information lines and information channels. The failures in commutation are accompanied by the disorders in the formation of cortical NT ICM magnetomes and impeded registration of IIMs on the pia.

Regression of cortical swelling and restored conductance through ICMs of the cortex results in restoration of tissue magnetomes, thus restoring extraneural processing of information in the brain and awareness. The period after 
the injury and before restoration of the record on the pia will be anterograde amnesia. If some part of the pia was injured (micro hemorrhages under the pia, ischemic foci, vessel damage, damage to the pia), events on the pia previously recorded become impossible to reproduce, which is frequently manifested in retrograde amnesia. So, conventional neurology and neurosurgery explains consciousness and memory disorders in brain injury as disordered conductance in the brain, but how this happens is not as yet clear, whereas information-commutation theory offers an explanation as to the development of consciousness and memory disorders of different severity.

The proposed theory explains the phenomenon of "distributed memory" described by Pribram and Lashley ${ }^{18}$ when they removed large portions of rat brains and found that doing this did not influence the rats' memory. If we accept that memories are carried by the pia that surrounds the brain, and not the neural network where the memories are supposed to be kept in neural network genes or in a protein network of neurons, it becomes clear that the IIMs of rat behavior formed as a result of training and labyrinth-passing skills are recorded on the remaining part of the pia, and these very parts are being used despite substantial removal of brain matter. Cognition, training, and education appear to be the result of the development and registration of the IIM holograms on the pia which is further algorithmized on the arachnoid and recorded on different parts of the pia. So even elimination of a considerable part of the brain NT will not affect the behavior patterns of animals and humans, because the long-term memory is recorded on the pia and not in the NT. Memory is the basic function of the pia in the brain. However, we cannot exclude a molecular (protein, genome, transcriptome) mechanism of memory reinforcement completely at this stage. Presumably, information relationships between the pia and NT ICMs require indepth research, and the role of protein memory, DNA and RNA, can be significant in the formation of short-term memory. As far as, the pia mater is a unique anatomical substrate for the recording, storage, and reproduction of information in a human body, the information is recorded, stored, and reproduced on the pia, and not in brain neural networks. This also explains the astonishing but so far unexplained observation of preserved memories in patients after clinical death when the energetic potential of the cerebral cortex (isoline on electroencephalography) and hippocampus is zeroed, and yet if such a patient recovers, they recognize their relatives and friends and even remember the events preceding their clinical death. If the memories were stored in the hippocampus, cortical neural circuits, or "field forms of substance", they would be completely "erased" upon clinical death, given that this state is characterized by complete absence of bioelectrical activity in brain tissue and disintegration of magnetoencephalographic manifestations in the brain.

Diffuse adhesion of blood elements on the pia causes failures in memorizing current information and disorders in the restoration of past information in the acute stage. Swelling of the brain parenchyma and cortex leads to disorders of current memorizing associated with edema of the pia. If blood elements in the SAS and SDS are not lysed, eg, blood clots are formed, scars and adhesions develop between the arachnoid and pia. Clinically, these would manifest as intellectualmnestic disorders in chronic TBI cases. They can deteriorate due to deformation of the pia and disorders of its holographic properties, ie, structural distortions of available holographic imprints of memory engrams on the pia. An outdated method called pneumoencephalography that was used to diagnose arachnoiditis and adhesions of cerebral meninges by air or gas injections into SAS, or, occasionally, to separate adhesions between the pia and the arachnoid, was extremely traumatic for the pia and for the long-term memory, and our theory explains why the procedure led to the loss of memory.

Subarachnoid and subdural hematoma in TBI lead to a whole range of psychopathological syndromes, varying from slight disorders of understanding and thinking to deep coma. Micro hemorrhage into the brain or white matter resulting from even slight brain injury (concussion) entails swelling of the tissue components of information channels and information lines (information channel error) around the hemorrhage focus, and disordered information signal conductance by input and output channels, manifesting in thinking difficulties and severe asthenia. Proposed brain ICO explains the nature of these symptoms as global disorders in the formation of holographic images in the SAS that can lead to severe disorders of the commuting capacities of cortical NT. Thus, it can be argued that any subarachnoid or subdural hematoma must be removed immediately, and independently of the size and type of compression, a hematoma under the pia may not be left. The consequences of its preservation in the brain will be much more significant than the potential complications from the surgery. This radically changes the existing strategies for surgical interventions in TBI. In the event of a subarachnoid hemorrhage, it is extremely important to organize evacuation of blood elements from the SAS, by cytapheresis of the CSF for example, thus avoiding the severe complications of TBI and memory loss.

Clinical comprehension of the pia as being the main information carrier of memory in the biological supercomputer of the brain and its main resource that provides for 
manipulations with memories manipulations gives a novel understanding of memory disorders or complete amnesia in transitory ischemic attacks and stroke. According to brain information theory, a disorder of blood supply in the vascular pool leads to an ischemic focus in brain NT and angiospasm in the area of the pia. An angiospastic or angioparalytic state of the arterioles of the pia in a cerebrovascular crisis leads to deformation and inability to reproduce information from the pia, and the situation deteriorates further as a result of disordered information conductance along input and output channels. The mechanism of such memory disorder seems obvious and allows development of novel therapy to treat mnestic disorders.

The mechanism of global amnesias developed after minor doses of clonidine, an antihypertensive agent, have always seemed unclear. Clonidine [2-(2.6-dichlorophenylamino)-2imidazoline] used to be widely used in the criminal sphere and and hundreds of the people with full amnesia stay in psychiatric hospitals for a very long time. Clinically, their organs and systems function normally and their encephalograms are within normal range, but their mnestic functions in the brain are lost, while intellectually intact. It would be interesting to study chronic memory disorders such as global amnesia and coma in clonidine overdose. Clonidine is an antihypertensive drug that specifically affects neurogenic regulation of vascular tone. To date, no research has offered a valid explanation regarding the psychopathological disorders and long-term and sometimes irreversible memory loss caused by the drug. However, brain information theory can explain the memory loss and comatose state associated with clonidine. Clonidine overdose mostly affects the pia mater, which has considerable vasculature and vegetative innervations, and not the brain NT or its major vessels. Angioparalysis of the pia causes it to sag (shrink), leading to coma and amnesia due to an inability to register information and disordered geometry of surface hologram membrane of the pia.

Changes in the geometry and elastic properties of the pia explain the toxic effect of alcohol abuse on memory. Initially, alcohol causes vasodilatation of vessels in the pia, straining it and leading to extremely precise record of the events; it then causes spasm of the pia vessels, leading to loss of memory for events associated with the alcohol abuse. A similar explanation of memory loss can be given for the symptoms of atrophic degeneration associated with atherosclerosis in the brain cortex. Atherosclerosis of small branches of the pia makes its vessels rigid, interfering with its plasticity (we forget names, words, telephone numbers, but can remember with effort) and progression of atrophic processes leads to degeneration of cortical neural layers closely connected with the pia, causing distortion of this delicate "hard drive" of our memory. Such initial manifestations of cerebral sclerosis as staggering and unstable gait result from the dislocated electromagnetic waves during the formation of the reflex arc of motor analyzer. Senile degenerative processes in the brain cortex are accompanied by the psycho-organic syndrome of Walther-Buel's triad, comprising poor memory, decreased understanding, and affect instability. Clinical neurology and psychiatry explains the psycho-organic syndrome in this pathology by atrophic processes in brain NT, although giving no explanations of how it forms. ICO of the brain explains the development of such symptoms by considerable expansion of the CSF space between the arachnoid and the pia in the projection of the frontal lobes in the systemic atrophic process. Under such circumstances, the diffusion patterns of magnetome electromagnetic waves of cortical ICMs and formation of holograms are disordered, as well as their localization on the arachnoid and registration on standard ICM-receivers and the pia, leading to weakened memory, understanding, and instability. We can force ourselves to restore forgotten information, but it is not remembered by itself. We can consciously control our gait, but usual gait which is based on reflexes is difficult. That is, we can intellectually control the conditioned reflex activity of the brain and are able to activate mechanisms of walking, memory, and attention. Interconnection of the frontal cortex with the limbic system of the brain is well known, and disorders at the level of channel information exchange can be accompanied by emotional disorders, thus explaining the development of affective disorders and emotional lability.

Currently, hereditary or acquired genetic defects have been detected in every neurological and psychiatric diseases. However, according to the proposed brain informationcommutation theory, genomic mutations have their manifestations in the transcriptome of nerve cells, ie, in all signal transduction pathways of an NT cell and in the cell proteome, so that they change the phenotype of affected nerve cells and change the wave and field processes in NT. This opens up a whole range of novel post-genome methodological approaches to the diagnosis and treatment of nervous and mental diseases. The therapies can involve correction of the proteome profile of NT by peptide engineering, or targeted change of the NT magnetic field (inductome), ie, a change in magnetization of pathological NT or cell magnetomes. New therapeutic opportunities for mental diseases can be found in magnetic resonance imaging-directed changes in brain tissue magnetization, according to the theory of brain 
ICO perceived as organic (genome-post-genome) diseases of information-communicative properties of NT ICMs in the brain, and not as mental or personality disorders.

The mechanisms and principles of brain operation put forward here may explain the development of Alzheimer's disease. Disorders at the level of the genome for cortical neurons lead to disorders in the transcriptome and proteome of NT ICM receiver of information output line and/or NT ICM-subscriber of information input line. Accumulation of amyloid in cortical neurons is a standard manifestation of an imbalance in neuron proteome profiling. A standard channel mistake is formed, and the conductance along cortical NT ICMs, and consequently the regulatory principle of brain operation, becomes disordered. Genetic damage makes the neurons of the NT ICM unable to compensate and restore independently, leading to severe disagreement of information transfer along output and input information lines, hologram record defects, and global mnestic disorders of the pia. In the attempt to restore proteome balance in NT ICMs, the neurons release excessive protein. The easiest way to restore the intracellular proteome and genome balance in the injured neuron is simple division of the cell in order to eliminate its pathological components.

Therefore, Alzheimer's disease is not a true disease of the brain, but a primitive way of compensation of neurons with a disordered proteome profile. Consequently, the neurons prepare to divide, get rid of unnecessary synaptic contacts, and as a result, become the reason for disintegration of cortical NT ICMs and then of the whole nervous system at the level of cortical NT ICMs due to crude information disorders of information reception/transfer. This concept could radically change the methodology of treatment for this disease.

Brain information-commutation theory sheds light on the mechanisms of hallucination and the range of mental diseases involving metabolic disorders of the brain, reactive psychosis, and schizophrenia. Hallucinations are formed IIMs that did not find complete materialization on definite cortical NT ICMs in corresponding analyzers owing to commutating errors of various brain regions, ie, IIMs materialized on other incidental analyzers. Disorders of algorithmization and programming of IIMs in the arachnoid are an explanation for delusional disorders. And, most important, it becomes clear why the outcome of the most of mental diseases is psychoorganic syndrome and dementia that result from disordered data conductance along NT ICMs.

Comprehensive analysis of genome-post-genome and biophysical disorders of brain ICM information levels can clarify the formation of hallucinations and psychopathological syndromes. Modeling such diseases with the discovered principles of brain operation and structure will open up a new era in the treatment of nervous and mental diseases.

\section{Conclusion}

These examples were provided to demonstrate novel approaches to the understanding of symptoms of nervous and mental diseases as viewed from the angle of brain ICO and theoretical neurology. However, this methodology can be extensively used in the analysis of clinical syndromes of nervous and mental illnesses, as well as in forensic medicine. This approach to brain ICO would provide the foundation for the artificial brain, artificial intelligence, and neurointerface development and form the basis for neurorobotics and neurocomputing. For more details of the theory, see web pages http://www.neurovita.ru and http://www.braintheory.ru.

\section{Disclosure}

The author reports no conflicts of interest in this work.

\section{References}

1. The Human Brain Project. A Report to the European Commission. Lausanne, Switzerland: The HBP-PS Consortium; 2012. Available from: https://www.humanbrainproject.eu/documents/10180/17648/ TheHBPReport_LR.pdf/18e5747e-10af-4bec-9806-d03aead57655. Accessed November 30, 2014.

2. Markram H. The Blue Brain Project. Nat Rev Neurosci. 2006;7: 153-160.

3. Marcus DS, Harwell J, Olsen T, et al. Informatics and data mining tools and strategies for the human connectome project. Front Neuroinform. 2011;5:4.

4. Brain Research through Advancing Innovative Neurotechnologies Working Group. Interim Report. National Institutes of Health; 2013. http://www.nih.gov/science/brain/. Accessed April 22, 2013.

5. Ukhtomsky AA. [Dominance as Working Principle of Nervous Centers]. Leningrad, Russia: Nauka; 1978. Russian.

6. Khomskaya ED. [Neuropsychology]. Moscow, Russia: Izdatelstvo Moskovskogo Universiteta; 1987. Russian.

7. Karlov VA. [Neurology guide for doctors]. In: Schtulman DR, Levin OS, editors. [Neurology: Manual for the Practicing Doctor]. 8th ed. Moscow, Russia: MEDpress-inform; 2008. Russian.

8. Bryukhovetskiy AS. [Clinical Oncoproteomics: Personalized Cancer Cell Therapy]. Moscow, Russia: Poligraf Plus; 2013. Russian.

9. Anokhin PK. [Biology and Neurophysiology of the Conditioned Reflex]. Moscow, Russia: Meditsina; 1968. Russian.

10. Sudakov KV. [Functional systems]. Moscow, Russia: Izdatelstvo RAMN; 2011. Russian.

11. Sandrigailo LI. [Anatomic Clinical Atlas in Neuropathology]. Minsk, Russia: Vysheishaya Shkola; 1978. Russian.

12. Duus P. [Topical Diagnosis in Neurology: Anatomy, Physiology, Signs, Symptoms]. Moscow, Russia: Vasar-Ferro; 1995.

13. Hecht-Nielsen R. A theory of thalamocortex. In: Hecht-Nielsen R, McKenna T, editors. Computational Models for Neuroscience. Berlin, Germany: Springer; 2003.

14. Baev KV. A new conceptual understanding of brain function: basic mechanisms of brain-initiated normal and pathological behaviors. Crit Rev Neurobiol. 2007;19:119-202.

15. Strangman G, Heindel WC, Anderson JA, Sutton JP. Learning motor sequences with and without knowledge of governing rules. Neurorehabil Neural Repair. 2005;19:93-114. 
16. Favorov O. The cortical pyramidal cell as a set of interacting error backpropagating dendrites: mechanism for discovering nature's order. In: Hecht-Nielsen R, McKenna T, editors. Computational Models for Neuroscience. Berlin, Germany: Springer; 2003.

17. Zemel RS, Mozer MC. Localist attractor networks. Neural Comput. 2001;13:1045-1064

18. Pribram K. Languages of the Brain: Experimental Paradoxes and Principles in Neuropsychology. 2nd ed. Monterey, CA, USA: Brooks/ Cole; 1977.

19. Routtenberg A. Lifetime memories from persistently supple synapses. Hippocampus. 2013;23:202-206.

20. Taylor JG, Taylor NR. Analysis of recurrent cortico-basal gangliathalamic loops for working memory. Biol Cybern. 2000;82:415-432.

21. Kandel ER. The molecular biology of memory storage: a dialog between genes and synapses. Biosci Rep. 2004;24:475-522.

22. Ramirez S, Liu X, Lin PA, et al. Creating a false memory in the hippocampus. Science. 2013;341:387-391.

23. Cauller L. The neurointeractibe paradigm: dynamical mechanics and the emergence of higher cortical function. In: Hecht-Nielsen R, McKenna T, editors. Computational Models for Neuroscience. Berlin, Germany: Springer; 2003.

24. Taylor JG. On the neurodynamics of the creation of consciousness. Cogn Neurodyn. 2007;1:97-118.

25. Capolupo A, Freeman WJ, Vitiello G. Dissipation of 'dark energy' by cortex in knowledge retrieval. Phys Life Rev. 2013;10:85-94.

26. Başar E, Başar-Eroglu C, Karakaş S, Schürmann M. Gamma, alpha, delta, and theta oscillations govern cognitive processes. Int $J$ Psychophysiol. 2001;39:241-248.

27. Neftci E, Binas J, Rutishauser U, Chicca E, Indiveri G, Douglas RJ. Synthesizing cognition in neuromorphic electronic systems. Proc Natl Acad Sci U S A. 2013;110:E3468-E3476.

28. Friston K, Kilner J, Harrison L. A free energy principle for the brain. J Physiol Paris. 2006;100:70-87.

29. Friston K. A free energy principle for biological systems. Entropy. 2012;14:2100-2121.

30. Friston KJ, Stephan KE. Free-energy and the brain. Synthese. 2007;159: 417-4458.

31. Just MA, Varma S. The organization of thinking: what functional brain imaging reveals about the neuroarchitecture of complex cognition. Cogn Affect Behav Neurosci. 2007;7:153-191.

32. Freeman WJ. Definitions of state variables and state space for braincomputer interface: Part 1. Multiple hierarchical levels of brain function. Cogn Neurodyn. 2007;1:3-14.
33. Freeman WJ. Understanding perception through neural "codes". IEEE Trans Biomed Eng. 2011;58:1884-1890.

34. Natarajan R, Huys QJ, Dayan P, Zemel RS. Encoding and decoding spikes for dynamic stimul. Neural Comput. 2008;20:2325-2360.

35. Constantinople CM, Bruno RM. Deep cortical layers are activated directly by thalamus. Science. 2013;340:1591-1594.

36. Beck F, Eccles JC. Quantum aspects of brain activity and the role of consciousness. Proc Natl Acad Sci U S A. 1992;89:11357-11361.

37. Jibu M, Yasue K. Quantum Brain Dynamics and Consciousness. An Introduction. Amsterdam, The Netherlands: John Benjamins Publishing Company; 1995.

38. Keppler J. A new perspective on the functioning of the brain and the mechanisms behind conscious processes. Front Psychol. 2013;4:242.

39. Roy A. Connectionism, controllers, and a brain theory. IEEE Trans Syst Man Cybern A Syst Hum. 2008;38:1434-1441.

40. Sanz Leon P, Knock SA, Woodman MM, et al. The Virtual Brain: a simulator of primate brain network dynamics. Front Neuroinform. 2013;7:10.

41. Stewart TC, Choo X, Eliasmith C. SPAUN: a perception-cognitionaction model using spiking neurons. Presented at the 34th meeting of the Cognitive Science Society, Sapporo, Japan, August 1-4, 2012.

42. Wong TM, Preissl R, Datta P, et al. IBM Research Report 1014, 2012. http://www.modha.org/blog/SC12/RJ10502.pdf. Accessed December 20, 2014

43. Crick F. Thinking about the brain. Sci Am. 1979;241:219-232.

44. Neumark YI. [Mathematical models of natural sciences and engineering (lectures)]. 3d ed. Nizhniy Novgorod, Russia: Izdatelstvo NNGU; 1997. Russian.

45. Hawkins J. How brain science will change computing. TED, 2003. Available from: http://www.ted.com/talks/jeff_hawkins_on_how_brain_ science_will_change_computing.html. Accessed July 12, 2012.

46. Devyatkov ND, Golant MB, Betsky OV. [Millimeter Waves and Their Role in the Processes of Life]. Moscow, Russia: Radio i Svyaz; 1991. Russian.

47. Gapeev AB. [Physical and Chemical Effects of Electromagnetic Radiation of Extremely High Frequencies at Cellular And Organism Levels]. Pushchino, Russia: Institut Biofiziki Kletki; 2006. Russian.

48. Bryukhovetskiy AS. [Cell Technologies in Neurooncology: Cytoregulatory Therapy of Brain Glial Tumors]. Moscow, Russia: Izdatelskaya Gruppa RONC; 2011. Russian.
Journal of Neurorestoratology

\section{Publish your work in this journal}

The Journal of Neurorestoratology is an international, peer-reviewed, open access online journal publishing original research and review articles on the subject of Neurorestoratology. To provide complete coverage of this revolutionary field the Journal of Neurorestoratology will report on relevant experimental research, technological advances, and

\section{Dovepress}

clinical achievements. The manuscript management system is completely online and includes a very quick and fair peer-review system, which is all easy to use. Visit http://www.dovepress.com/testimonials.php to read real quotes from published authors. 